\title{
Minimización de los efectos atmosféricos en el índice espectral de la vegetación IVIS
}

\section{Minimization of atmospheric effects on the ISVI spectral vegetation index}

\author{
Fernando Paz Pellat ${ }^{1:}$
}

${ }^{1}$ GRENASER, Colegio de Postgraduados. Carretera México-Texcoco km 36.5. 56230 Montecillo, Texcoco, México.
${ }^{\ddagger}$ Autor responsable (ferpazpel@gmail.com)

\section{RESUMEN}

Existe una importante necesidad de minimizar los efectos atmosféricos en la información espectral de los sensores remotos en plataformas espaciales, para evitar subestimaciones de variables biofísicas que se asocian con los datos de imágenes satelitales. En el presente trabajo se desarrolló un algoritmo genérico, con base en argumentos teóricos sólidos, para analizar series temporales del índice espectral de la vegetación IVIS (índice de vegetación basado en curvas iso-suelo) y evitar así los problemas asociados al diseño clásico de índices de vegetación, en los que la señal espectral se satura rápidamente. Los resultados al aplicar el algoritmo a series temporales de píxeles de imágenes satelitales del sensor AVHRR mostraron que se logró la reducción y estandarización de los efectos atmosféricos en el IVIS. El uso de los valores máximos del IVIS en una serie temporal (ventanas de muestreo), permitió tener una aproximación razonable a condiciones atmosféricas de efectos mínimos o estandarizados. En conclusión, aún cuando el esquema que se planteó no logró eliminar en su totalidad el efecto atmosférico con el IVIS, sí lo redujo hasta valores mínimos. El algoritmo que se desarrolló fue lo suficientemente simple para su uso operacional, en comparación con los métodos de corrección atmosférica que utilizan inversiones de modelos atmosféricos.

Palabras clave: curvas iso-suelo, subestimaciones biofísicas, efecto suelo-atmósfera, curva paramétrica del crecimiento.

\section{SUMMARY}

It is essential to minimize atmospheric effects on spectral information of remote sensors from space platforms to avoid under estimation of biophysical variables associated with satellite image data. In this paper, a generic algorithm was developed, based on sound theoretical arguments, to analyze time series ISVI spectral vegetation index (vegetation index based on iso-soil curves), thus avoiding the problems associated with the classic design of vegetation indices, where the spectral signal saturates quickly. The results, when applying the algorithm in pixel time series of AVHRR satellite images, showed that reduction and standardization of atmospheric effects in the ISVI was achieved. Using ISVI maximum values in time series (temporal window), a reasonable approximation to atmospheric conditions with minimum or standardized effects was obtained. In conclusion, although the scheme developed failed to eliminate the atmospheric effect on ISVI entirely, it was reduced to a minimum. The algorithm developed was simple enough for operational use, with regard to atmospheric correction methods using radiative model inversions.

Index words: iso-soil curves, biophysical underestimations, soil-atmosphere effect, parametric growth curve.
Cita recomendada:

Paz Pellat, F. Minimización de los efectos atmosféricos en el índice espectral de la vegetación IVIS. Terra Latinoamericana 36: 31-38.

DOI: https://doi.org/10.28940/terra.v36i1.230
Recibido: enero de 2017. Aceptado: noviembre de 2017. Publicado en Terra Latinoamericana 36: 31-38: 


\section{INTRODUCCIÓN}

La atmósfera distorsiona la señal que reciben los sensores remotos que se ubican en las plataformas espaciales, lo que produce una subestimación de las variables biofísicas que se asocian con la vegetación, tales como el índice de área foliar (IAF) y la cobertura aérea de la vegetación (fv); de tal manera que, si no hay una minimización de la distorsión, las estimaciones que se derivan de datos satelitales tienen una incertidumbre muy alta (Huete y Liu, 1984).

Comúnmente, el uso de la información espectral de imágenes satelitales se lleva a cabo a través de la construcción de índices de vegetación (IV) que se relacionan con características como el IAF, la fv, las propiedades ópticas del follaje y del suelo, entre otras (Ross, 1981), cuya determinación se basa en las diferencias de contraste entre una banda espectral fotosintética como el rojo (R) y una no fotosintética como el infrarrojo cercano (IRC). Para reducir el efecto de la atmósfera en los IV, se pueden corregir las reflectancias de las bandas espectrales de los IV o hacer una modificación directa de éstos (Kaufman y Tanre, 1996).

El diseño más común de los IV consiste en la minimización del efecto del fondo, es decir, del suelo debajo el cultivo (Huete, 1988), lo cual es particularmente importante para vegetación con cobertura aérea parcial del suelo. En este sentido, el NDVI o índice de vegetación de la diferencia normalizada (Rouse et al., 1974) es el que más se utiliza, aún cuando tiene severas limitaciones (Paz et al., 2008). Desde una perspectiva más genérica, el diseño de un IV debe cumplir varios objetivos en forma simultánea; especialmente, minimizar los efectos del suelo y de la atmósfera, que suelen ser interactivos (Huete y Liu, 1984); tal es el caso del Índice de Vegetación Mejorado o EVI (Huete et al., 1999), cuyo desarrollo se basó en el trabajo de Liu y Huete (1995). El problema de este esquema de minimización de efectos conjuntos es que resulta adecuado únicamente para las condiciones de optimización predeterminadas. Otro ejemplo es el índice NDVIcp (Paz et al., 2008) que se optimizó para un aerosol genérico y un modelo atmosférico (Paz et al., 2008), pero no resulta adecuado para otros aerosoles o atmósferas. Por lo tanto, para evitar este tipo de restricciones, es necesario desarrollar una estrategia global de minimización de efectos atmosféricos, no dependiente del tipo de aerosol o del modelo atmosférico.

Por lo que respecta al diseño de IV con reducción del efecto del suelo, la mayoría de los índices se basan en las líneas iso-IAF (igual vegetación, diferentes propiedades ópticas del suelo) del espacio del R-IRC (Gilabert et al., 2002), lo que provoca problemas al aproximar la etapa vegetativa completa de crecimiento de la vegetación (Paz et al., 2008). Romero et al. (2009) presentaron un índice que modela adecuadamente toda la etapa de crecimiento (aún con problemas de saturación de la señal espectral), pero que requiere de constantes empíricas por cultivo o tipo de vegetación. Una alternativa a este esquema de modelación es usar las curvas iso-suelo (igual suelo y vegetación variable) del espacio del R-IRC, como el índice de vegetación que se basa en curvas iso-suelo (IVIS), desarrollado por Paz et al. (2011), el cual reduce los efectos del suelo a nivel de píxel en una imagen satelital.

Con base en lo anterior, el objetivo del presente trabajo fue desarrollar una aproximación general al problema de minimización conjunta del efecto atmosférico y de fondo (generalmente suelo) bajo la vegetación, para el índice IVIS, a través de la generación de un algoritmo que se revisa con datos satelitales del sensor AVHRR en las plataformas espaciales NOAA (NOAA-6 a NOAA-19).

\section{MATERIALES Y MÉTODOS}

Se desarrolló un algoritmo genérico, con fundamento en los argumentos teóricos que se detallan más adelante, que permitió el análisis de series temporales del índice espectral de la vegetación IVIS (índice de vegetación basado en curvas iso-suelo), con la finalidad de evitar todos aquellos problemas que se asocian al diseño clásico de índices de vegetación, tales como las líneas de igual índice de área foliar, en los que la señal espectral se satura rápidamente.

\section{Fundamento Teórico}

En la Figura 1a se muestran los patrones espectrales en el espacio del R-IRC que se asocian al incremento en el IAF para un cultivo de maíz, en el que el fondo lo constituyen suelos con diferentes propiedades ópticas. Los datos se generaron mediante el uso de simulaciones con modelos de radiación (Paz et al., 2005). En dicha 

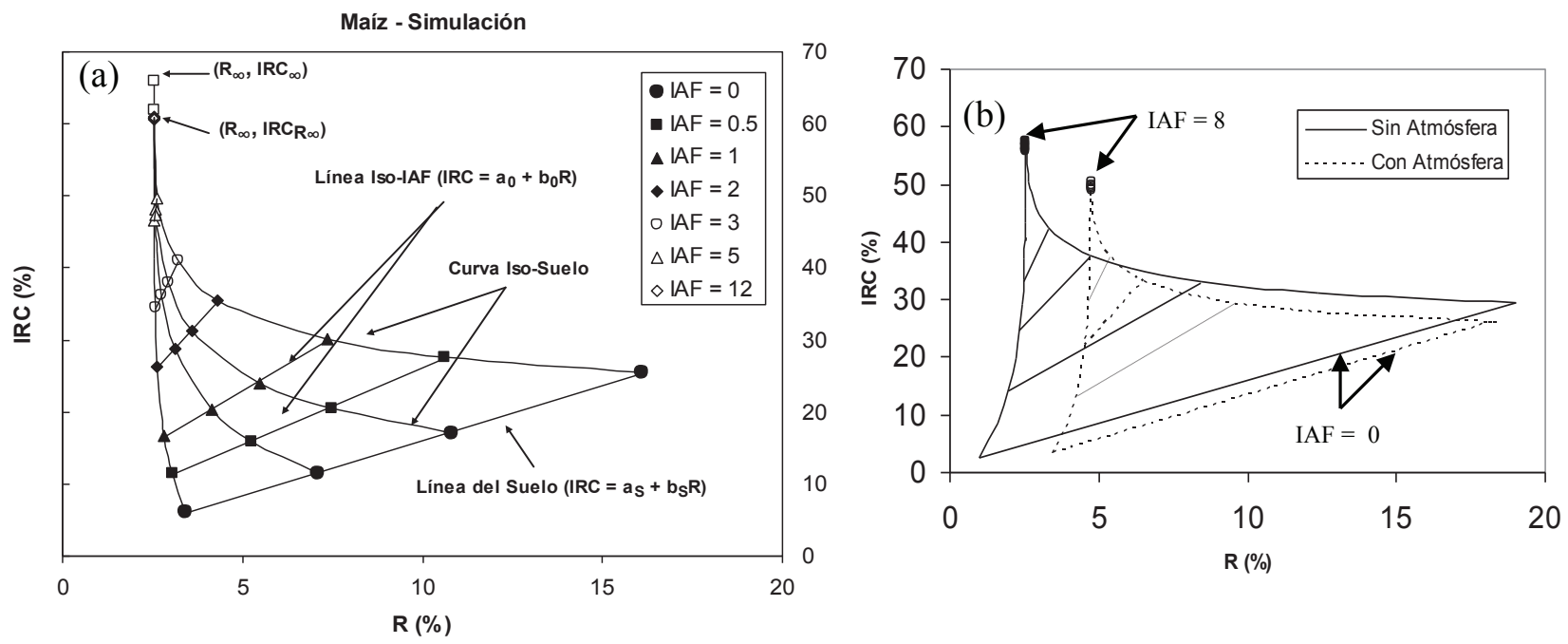

Figura 1. (a) Simulaciones de modelos de radiación en el espacio del R-IRC (reflectancias en porcentajes) asociadas al crecimiento de un cultivo de maíz; (b) efecto atmosférico en las simulaciones. IAF = índice de área foliar.

figura se observan las líneas iso-IAF, que van desde la línea del suelo (IRC $=\mathrm{a}_{\mathrm{S}}+\mathrm{b}_{\mathrm{s}} \mathrm{R}$; donde $\mathrm{a}_{\mathrm{s}} \mathrm{y} \mathrm{b}_{\mathrm{s}}$ son constantes), con líneas que cambian en función del IAF, hasta alcanzar un ápice superior que se define por las reflectancias en el infinito $(\mathrm{R} \infty$, IRC $\infty)$, las cuales se asocian a un medio denso donde sólo se observa la vegetación y no el suelo. Las curvas isosuelo de la Figura 1a se refieren al crecimiento de la vegetación con propiedades espectrales del suelo fijas. A nivel de pixeles individuales, el patrón espectral que corresponde al crecimiento de la vegetación queda entre un par de curvas iso-suelo (suelo seco y húmedo, por ejemplo) continuas (Figura 1a).

Por otra parte, en la Figura $1 \mathrm{~b}$ se muestran las líneas iso-IAF y las curvas iso-suelo (Paz et al., 2008), pero con reducción de sus valores a causa del efecto atmosférico. Dicho efecto distorsiona los patrones espectrales en el espacio del R-IRC, lo que produce subestimaciones en los IV y destaca la necesidad de minimizarlo. En la Figura 1b se observa, también, que los efectos atmosféricos hacen que las curvas iso-IAF se sitúen por debajo de aquellas con independencia de dicho efecto, por lo que cualquier índice que no subsane esta problemática, tenderá a subestimar el valor real del IAF, con las variables biofísicas que involucre.

El índice IVIS que desarrollaron Paz et al. (2011) con base en las líneas iso-suelo, se define por la siguiente Ecuación (1):

$$
\begin{aligned}
& I V I S=-\ln \left(\frac{d I R C \infty-d I R C}{d I R C \infty}\right) \\
& d I R C \infty=I R C \infty-\left(a_{S}+b_{S} R \infty\right) \\
& d I R C=I R C-\left(a_{S}+b_{S} R\right)
\end{aligned}
$$

En la Ecuación (1), los efectos atmosféricos sobre las bandas espectrales del R e IRC pueden aproximarse de Paz et al. (2006), como:

$$
\begin{aligned}
& R a=a_{R}+b_{R} R \\
& I R C a=a_{I R C}+b_{I R C} I R C
\end{aligned}
$$

donde la letra a en R e IRC implica efecto atmosférico. Las constantes $a$ y $b$ aproximan la radiancia de trayectoria y la transmitancia de doble vía en términos efectivos, de la atmósfera.

Paz et al. (2006) demostraron que la relación entre el dIRC con y sin atmósfera cumple con la Ecuación (3):

$\frac{d I R C a}{d I R C}=b_{I R C}$

donde el dIRCa tiene efectos atmosféricos y se define en función de la línea del suelo, con los mismos efectos. Así, de las Ecuaciones (1) y (3), se tiene que el IVIS es invariante bajo efectos atmosféricos:

$I V I S=I V I S a$ 
La Ecuación (4) es producto de que, en el caso del efecto atmosférico, todas las líneas iso-IAF, (incluida la del suelo) tienen las mismas variaciones en los parámetros $\left(a_{0} \mathrm{y} b_{0}\right) \mathrm{y}$, como el IVIS utiliza la línea del suelo como punto de partida, las métricas asociadas a éste permanecen similares al caso de sin atmósfera.

La invariancia, bajo efectos atmosféricos, precisa conocer los parámetros de las líneas del suelo con y sin atmósfera, por lo que resulta difícil de aplicar en términos operacionales en imágenes del sensor AVHRR (píxeles de 121 ha, a nadir). Un procedimiento alternativo para reducir los efectos atmosféricos en el IVIS, es usar una línea virtual del suelo similar para los casos con y sin efectos de la atmósfera. Así, con una línea del suelo virtual (cualquier valor de $a_{0} \mathrm{y} b_{0}$ que se defina) $\mathrm{IRC}=b_{\mathrm{S}} \mathrm{R}\left(a_{\mathrm{S}}=0\right)$ y las Ecuaciones (1) y (2) se puede definir a dIRC como:

$$
\begin{aligned}
& d I R C=I R C-b_{S} R \\
& d I R C a=a_{I R C}+b_{I R C}-b_{S} a_{R}-b_{S} b_{R} R
\end{aligned}
$$

Ahora bien, si se usa una línea iso-IAF, Figura 1a, la cual se define por:

$$
I R C=a_{0}+b_{0} R
$$

es posible sustituir esta ecuación en la relación de dIRC, Ecuación (1), y despejarla para R:

$$
R=\frac{d I R C-a_{0}}{b_{0}-b_{S}}
$$

La Ecuación (6) puede ahora sustituirse en la Ecuación (5) para poner dIRCa en función de dIRC y simplificarse como:

$$
\begin{aligned}
& d I R C a=S+T d I R C \\
& S=\left(a_{I R C}-b_{S} a_{R}\right)+\left[\frac{b_{S} a_{0}}{b_{0}-b_{S}}\right]\left(b_{R}-b_{I R C}\right) \\
& T=\left[\frac{b_{0} b_{I R C}-b_{S} a_{R}}{b_{0}-b_{S}}\right]
\end{aligned}
$$

De acuerdo con Paz et al. (2006 y 2008), se tienen las siguientes condiciones:

$$
\begin{aligned}
& a_{\mathrm{R}}>a_{I R C} \\
& b_{\mathrm{R}}<b_{I R C} \\
& b_{0} \geq b_{S} \\
& 0<b_{\mathrm{R}} \leq 1 \\
& 0<b_{I R C} \leq 1
\end{aligned}
$$

Por lo general, $b_{\mathrm{S}}>1$ (Baret et al., 1983), aunque puede ser necesario considerar un parámetro virtual con $b_{\mathrm{S}} \leq 1$ para la situación de píxeles con poca vegetación.

De las relaciones (8) y (9) se tiene que $\mathrm{S}<0$ $\left(a_{0} \geq 0\right)$; así, para evaluar a $\mathrm{T}$ se pueden usar las relaciones entre $b_{R}$ y $b_{\text {IRC }}$, tal como se muestra en la Figura 2, que se obtuvo de simulaciones con modelos de radiación (Vermote et al., 1997) con un ángulo cenital solar $\theta \mathrm{s}=0$, altitud $=0$, atmósfera tropical y aerosol continental y, en la que cada punto representa un espesor óptico atmosférico o visibilidad.

A partir de los patrones que determinaron $\mathrm{Paz}$ et al. (2008) para la relación entre $b_{\mathrm{R}} \mathrm{y} b_{\mathrm{IRC}}$, la Figura 3 muestra $b_{\mathrm{R}} / b_{\mathrm{IRC}}$ con un espesor óptico atmosférico a $550 \mathrm{~nm}\left(\tau_{550}\right)$, atmósfera tropical y aerosoles continental, desértico y de quema de biomasa (aerosol genérico), característicos de la aplicación de los sensores remotos en el monitoreo de la vegetación.

En la Figura 3, el aerosol genérico representa convenientemente los tres tipos de aerosoles y define el patrón en relación a la turbiedad o visibilidad de la atmósfera $\left(\tau_{550}\right)$. En la Figura 4, por otra parte, se muestra el caso de diferentes modelos atmosféricos con el aerosol genérico y se puede observar que

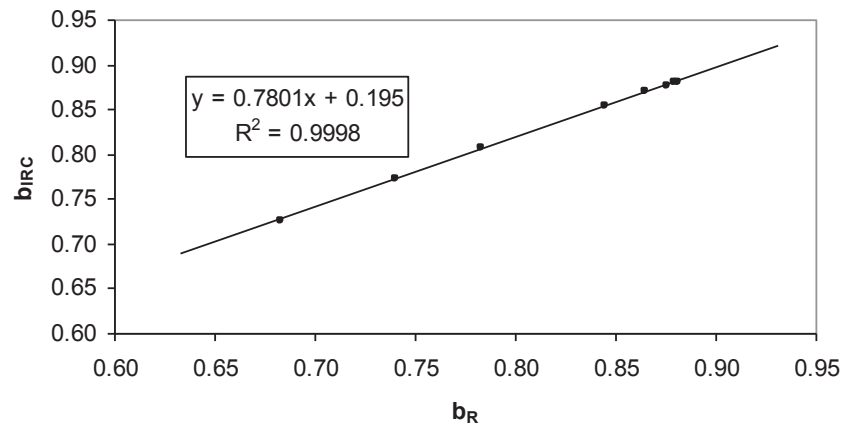

Figura 2. Relación entre $b_{\mathrm{R}} \mathrm{y} b_{\mathrm{IRC}}$ para simulaciones atmosféricas con modelos de radiación. 


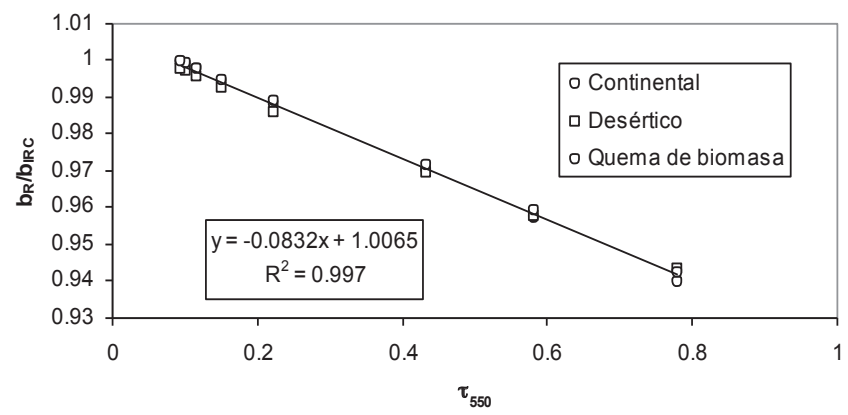

Figura 3. Relación entre $b_{\mathrm{R}} / b_{\mathrm{IRC}}$ y $\tau_{550}$ para una atmósfera tropical y un aerosol genérico (Paz et al., 2008).

las variaciones de $b_{\mathrm{R}} / b_{\text {IRC }}$, con la visibilidad atmosférica, se reducen para los modelos atmosféricos y aerosoles de interés, en la aplicación del monitoreo de la vegetación.

Para evaluar el parámetro T, Ecuación (8), como ejemplo se puede usar la relación de la Figura 2, con un valor de $b_{\mathrm{S}}=1$. En la Figura 5 se muestra la relación entre $b_{0}$ y $\mathrm{T}$ para diferentes visibilidades atmosféricas (valores de $b_{\mathrm{R}}$ ), misma que es discontinua en $b_{0}=b_{\mathrm{S}}$. Para valores de $b_{0}<b_{\mathrm{s}}, \mathrm{T}<1 \mathrm{y}$, para valores un poco mayores que $b_{\mathrm{s}}(1.3$ en la Figura 5$)$, se obtiene $\mathrm{T}<0$.

De acuerdo con análisis similares a los de la Figura 5, generalmente cuando $b_{0}>1.5$ se tiene que $\mathrm{T}<1$. Ahora bien, si se considera que las líneas reales del suelo (y de residuos o vegetación seca) tienen valores de $b_{\mathrm{S}}>1$, la condición de $b_{0}>1.5$ representa condiciones que típicamente se encuentran en esta situación para las líneas de igual vegetación (iso-IAF).

No obstante, lo que se discutió para el caso de $b_{\mathrm{S}}$, es recomendable utilizar un valor de $b_{\mathrm{s}}=0.2$ para tener mayor certeza de que $\mathrm{T}<1$.

La Figura 6 muestra la relación entre el índice de área foliar (IAF) con $\mathrm{a}_{0} \mathrm{y}_{0}$ para las simulaciones con modelos de radiación de la Figura 1a.

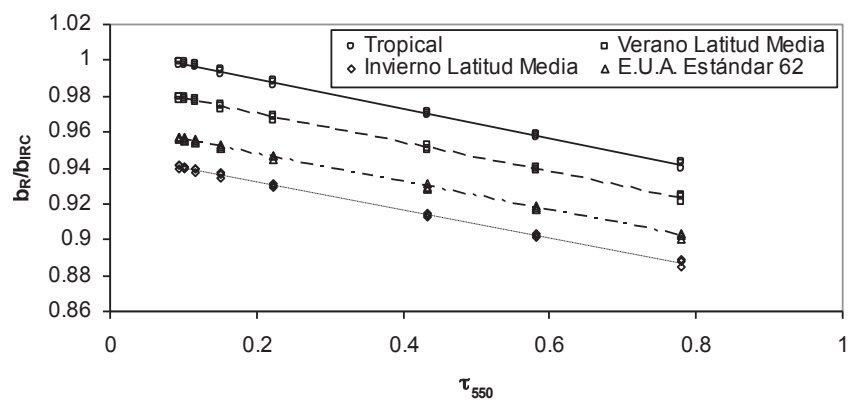

Figura 4. Relación entre $b_{\mathrm{R}} / b_{\mathrm{IRC}} \mathrm{y} \tau_{550}$ para diferentes modelos atmosféricos y un aerosol genérico (Paz et al., 2008).

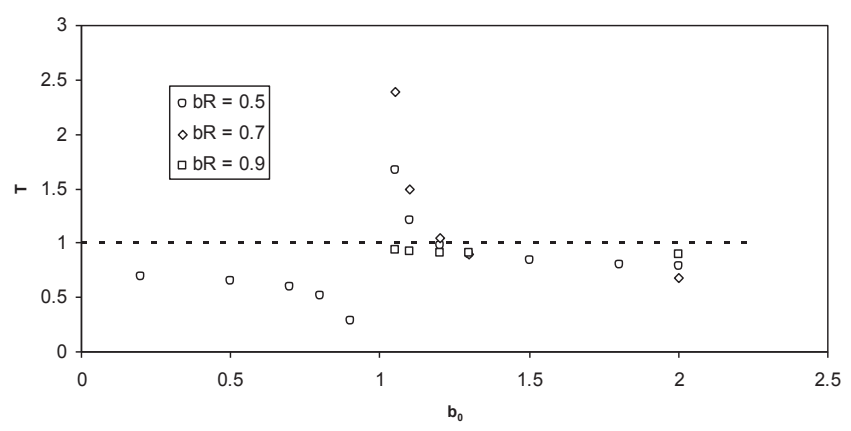

Figura 5. Relación entre $b_{0}$ y $T$ para diferentes visibilidades atmosféricas.

De acuerdo con la Figura 6, cuando la vegetación (medio físicamente homogéneo o medio turbio en términos radiativos) se torna más densa, el valor de $\mathrm{a}_{0}$ se vuelve negativo (Figura 6). Yoshioka et al. (2000) mostraron que para un medio heterogéneo generalmente $a_{0}>0$, pero si $b_{\mathrm{S}} a_{0} /\left(b_{0}-b_{\mathrm{S}}\right)$ en $\mathrm{S}$, Ecuación (8), con valores de $b_{\mathrm{S}}<1$, el caso de $a_{0}<0$ tiene un efecto muy reducido en el valor de éste $\left(b_{0}\right.$ crece casi al mismo ritmo que $\mathrm{a}_{0}$ decrece). La multiplicación del término que se discute por $\left(b_{\mathrm{R}}-b_{\mathrm{IRC}}\right)$ de $\mathrm{S}$, Ecuación (8), hace que la situación de $\mathrm{S}>0$ tenga una probabilidad muy baja, si es que se presenta.

Todo lo anterior implica, en lo general, que $\mathrm{S}<0$ y $\mathrm{T}<1$, Ecuación (8), con parámetros definidos de $b_{\mathrm{s}}$, por lo que dIRC $a<\operatorname{dIRC}$ (IVIS $a<$ IVIS). En el caso de nubes o de cuerpos de agua, se tiene que dIRC es negativo o cercano a cero, por lo que el IVIS es negativo o cercano a cero. La relación $\operatorname{dIRC} a<\mathrm{dIRC}$ puede generalizarse a una del tipo dIRC $_{2}<$ dIRC $_{1}\left(\right.$ IVIS $_{2}<$ IVIS $\left._{1}\right)$, donde $\mathrm{dIRC}_{2}$ representa un mayor efecto atmosférico que $\mathrm{dIRC}_{1}$. A partir de estas consideraciones, en una serie temporal del IVIS, los valores más grandes representan la condición de efectos atmosféricos mínimos, con cualquier IAF, por lo que se puede usar un algoritmo que seleccione estos valores máximos para el desarrollo de un modelo paramétrico del crecimiento de la vegetación; propuesta metodológica que permite la construcción de un algoritmo simplificado consistente en la selección de los valores máximos del IVIS en una ventana temporal deslizante de análisis.

Cabe destacar, que la presente propuesta difiere del método de usar valores máximos (MVC, maximum value composite) del índice NDVI en una ventana temporal (Holben, 1986; Kaufman y Tanre, 1996), que es un método que usa valores que se asocian a visiones 


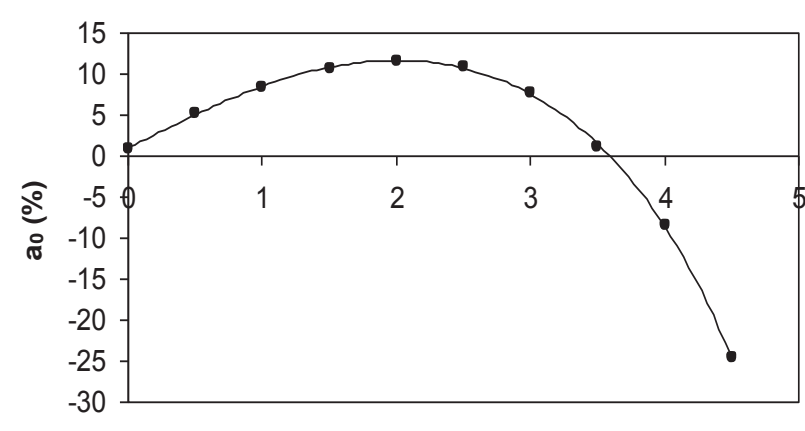

IAF

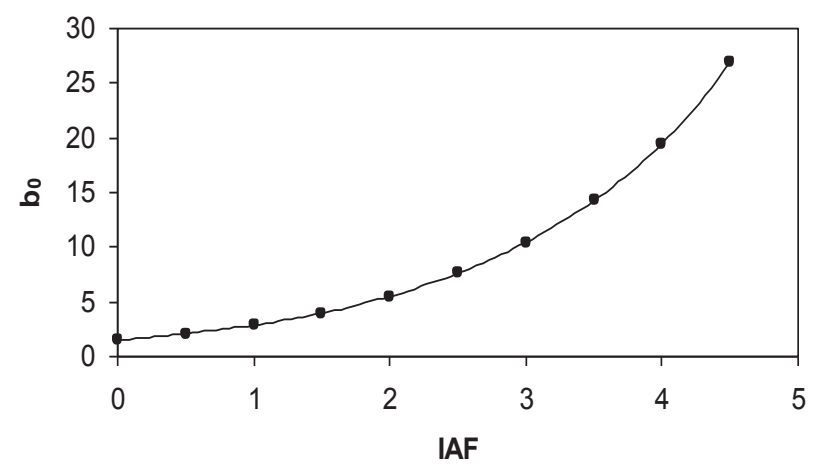

Figura 6. Relación entre el IAF con $a_{0}$ y $b_{0}$ de las simulaciones con modelos de radiación de la Figura 1a. IAF = índice de área foliar.

del sensor en ángulos cenitales cercanos a nadir y bajo la consideración de una correlación entre los efectos de los aerosoles y del vapor de agua. Además, se trata de un método específico al NDVI, cuyo uso se orienta a variaciones en la geometría sol-sensor. El método propuesto se diseñó en función de los parámetros de la línea del suelo, para asegurar que los valores máximos en una ventana temporal de observación correspondan con los de menor efecto atmosférico, independientemente de la geometría sol-sensor.

\section{RESULTADOS Y DISCUSIÓN}

Para analizar el efecto de $b_{\mathrm{s}}$ en el algoritmo de minimización atmosférica y obtener una primera aproximación de la curva paramétrica del crecimiento de la vegetación, se puede utilizar dIRC $\infty=100 \%$ (reflectancias en porcentajes) y $b_{\mathrm{s}}=1.0\left(a_{\mathrm{s}}=0\right)$, condiciones que resultan más o menos adecuadas para los patrones de crecimiento de diferentes ecosistemas (pastizales, matorrales y bosques/selvas).

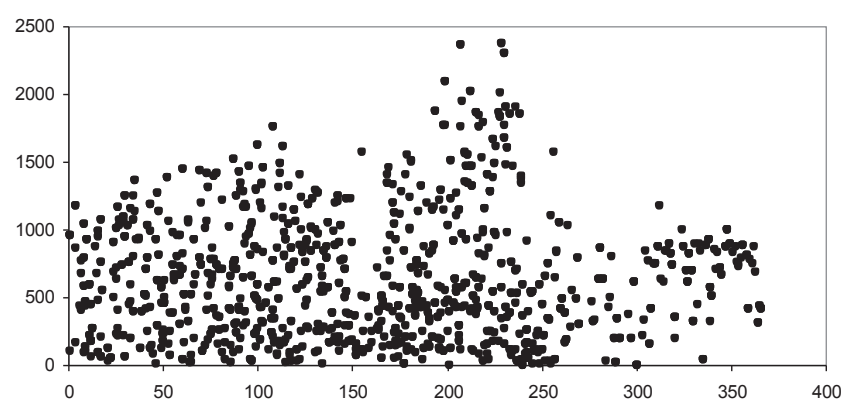

Figura 7. Patrón temporal del IVIS para el uso de varios satélites (sensor AVHRR) en el 2008, datos originales. En el eje X se representan días julianos y en el eje Y el valor IVIS multiplicado por 10000 .
En la Figura 7 se observa el patrón temporal para el año 2008 de los IVIS de un píxel en una selva de la Península de Yucatán, en México, para el que se usó el sensor AVHRR. La geometría sol-sensor se estandarizó (Bolaños et al., 2007; Bolaños y Paz, 2010) para reducir el impacto de este efecto. En dicha figura se destaca la gran dispersión en los valores de los IVIS, producto del uso de varios satélites NOAA para obtener más de un valor en un día, por lo que representa condiciones atmosféricas diferentes que pueden variar desde ausencia de nubes (valores más altos del IVIS) hasta presencia de nubes densas (valores cercanos a 0 del IVIS), esto para el efecto atmosférico. En condiciones sin atmósfera, los valores altos del IVIS corresponderían a vegetación densa y los valores bajos a vegetación escasa. La combinación de vegetación y atmósfera es interactiva.

En la Figura 8 se muestran los resultados que se obtuvieron con un algoritmo simple (ventana temporal de análisis), al seleccionar el valor máximo en una ventana móvil de 5 días (2 pasos). Se observa

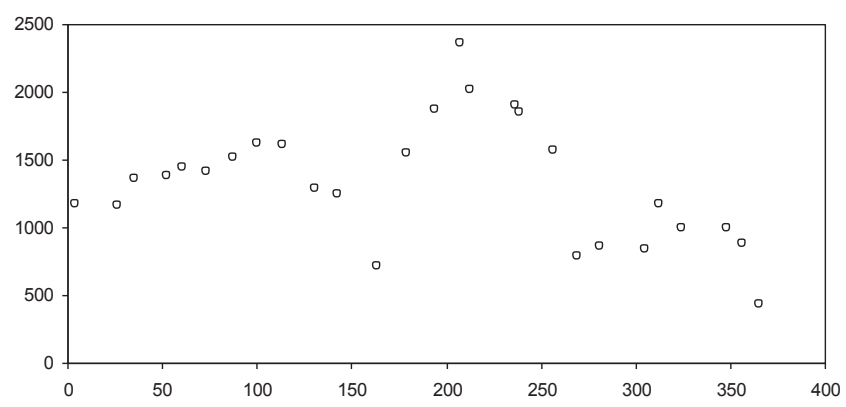

Figura 8. Patrón temporal del IVIS para el uso de varios satélites (2008), algoritmo aplicado de ventana móvil para selección de valores máximos del IVIS. En el eje $\mathrm{X}$ se representan días julianos y en el eje Y el valor del IVIS multiplicado por 10000. 
que el patrón que se esperaba de los IVIS (datos de experimentos en condiciones controladas de Paz et al., 2011), se despliega en términos más claros.

Cuando la disponibilidad de satélites es menor, con la misma vegetación y para datos de 1988, las Figuras 9 y 10 muestran tendencias similares a las de las Figuras 7 y 8, respectivamente, lo que denota que el algoritmo que se usó para caracterizar la evolución temporal de la vegetación es altamente robusto para su aplicación en términos operacionales.

Es importante destacar que el uso de simulaciones con modelos de radiación, con y sin efectos atmosféricos, para validar los desarrollos, está implícito en las Ecuaciones (8) y (1) por lo que no se considera necesario incluir esta evidencia.

A partir de la validación de los modelos de simulaciones con modelos de radiación de efectos atmosféricos de Vermote et al. (1997), se muestra que la aplicación del algoritmo a casos particulares de imágenes satelitales no aporta más información.

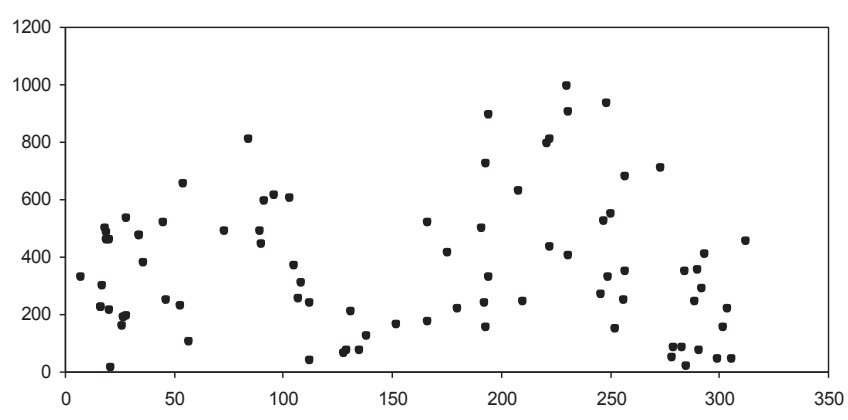

Figura 9. Patrón temporal del IVIS para el uso de varios satélites (1988), datos originales. El eje X representa días julianos y el eje Y el IVIS multiplicado por 10000.

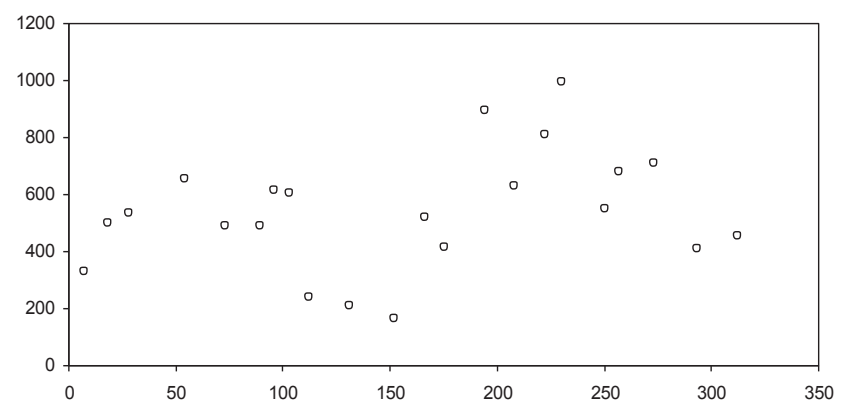

Figura 10. Patrón temporal del IVIS para el uso de varios satélites (1988), algoritmo aplicado. El eje $X$ representa días julianos y el eje Y el IVIS multiplicado por 10000.

\section{CONCLUSIONES}

- Se desarrolló un esquema que establece la condición dIRC $_{2}<$ dIRC $_{1}$ y, por lo tanto, IVIS $_{2}<$ IVIS $_{1}$, donde el subíndice 2 corresponde a la condición de mayor efecto atmosférico y 1 a la de menor, de tal manera que se logró la reducción y estandarización de los efectos atmosféricos en el índice de la vegetación con base en curvas iso-suelo o IVIS. El uso de los valores máximos del IVIS (menores efectos atmosféricos garantizados) en una serie temporal, permitió tener una aproximación razonable a condiciones atmosféricas de efectos mínimos o estandarizados.

- Aún cuando el esquema que se plantea no logra eliminar en su totalidad el efecto atmosférico en el IVIS, sí lo reduce hasta valores mínimos (dependientes de las condiciones locales y temporales de la atmósfera). El algoritmo operacional que se desarrolló (selección de valores máximos en ventanas temporales) fue lo suficientemente simple para su aplicación rutinaria.

\section{LITERATURA CITADA}

Baret, F., S. Jacquemoud, and J. F. Hanocq. 1983. The soil line concept in remote sensing. Remote Sens. Rev. 7: 65-82.

Bolaños G., M. A. y F. Paz P. 2010. Modelación general de los efectos de la geometría iluminación-visión en la reflectancia de los pastizales. Rev. Mex. Cienc. Pec. 1: 349-361.

Bolaños, M., F. Paz, E. Palacios, E. Mejía y A. Huete. 2007. Modelación de los efectos de la geometría sol-sensor en la reflectancia de la vegetación. Agrociencia 41: 527-537.

Gilabert, M. A., J. González, F. J. García, and J. Meliá. 2002. A generalized soil-adjusted vegetation index. Remote Sens. Environ. 82: 303-310.

Holben, B. N. 1986. Characteristics of maximum value composite images for temporal AVHRR data. Int. J. Remote Sens. 7: $1417-1434$.

Huete, A. R. 1988. A Soil-adjusted vegetation index (SAVI). Remote Sens. Environ. 25: 295-309.

Huete, A. R. and H. Q. Liu. 1994. An error and sensitivity analysis of the atmospheric-and soil-correcting variants of the NDVI for the MODIS-EOS. IEEE Trans. Geosci. Remote Sens. 32: 897-905

Huete, A., C. Justice, and W. van Leeuwen. 1999. MODIS Vegetation Index (MOD 13), Algorithm Theoretical Basis Document. NASA Goddard Space Flight Center. Greenbelt, MD, USA

Kaufman, Y. J. and D. Tanré. 1996. Strategy for direct and indirect methos for correcting the aerosol effect on remote sensing: From AVHRR to EOS-MODIS. Remote Sens. Environ. 55: 65-79. 
Liu, H. Q. and A. Huete. 1995. A feedback based modification of the NDVI to minimize canopy background and atmospheric noise. IEEE Trans. Geosci. Remote Sens. 33: 457-465.

Paz, F., E. Palacios, E. Mejía, M. Martínez y L. A. Palacios. 2005. Análisis de los espacios espectrales de la reflectividad del follaje de los cultivos. Agrociencia 39: 293-301.

Paz, F., E. Palacios, L. A. Palacios, L. Tijerina y E. Mejía. 2006. Correcciones atmosféricas usando patrones invariantes en el espacio del rojo e infrarrojo cercano. Rev. Latin. Rec. Nat. 2: 3-16.

Paz, F., E. Palacios, M. Bolaños, L. A. Palacios, M. Martínez, E. Mejía y A. Huete. 2007. Diseño de un índice espectral de la vegetación: NDVIcp. Agrociencia 41: 539-554.

Paz, F., E. Palacios, M. Bolaños, E. Palacios, L. A. Palacios, M. Martínez y A. Huete. 2008. Optimización del índice espectral de la vegetación NDVIcp. Agrociencia 42: 925-937.
Paz, F., M. Reyes y E. Medrano. 2011. Diseño de índices espectrales de la vegetación usando curvas iso-suelo. Agrociencia 45: 121-134.

Romero, E., F. Paz, E. Palacios, M. Bolaños, R. Valdez y A. Aldrete. 2009. Diseño de un índice espectral de la vegetación desde una perspectiva conjunta de los patrones exponenciales y lineales del crecimiento. Agrociencia 43: 291-307.

Rouse, J. W., R. H. Haas, J. A Schell, D. W. Deering, and J. C. Harlan. 1974. Monitoring the vernal advancement of retrogradation of natural vegetation. MASA/GSFC. Type III. Final Report. Greenbelt, MD, USA.

Vermote, E. F., D. Tanré, J. L. Deuzé, M. Herman, and J. J. Morcrette. 1997. Second simulation of the satellite signal in the solar spectrum, 6S: An overview. IEEE Trans. Geosci. Remote Sens. 35: 675-686.

Yoshioka, H., T. Miura, A. R. Huete, and B. D. Ganapol. 2000. Analysis of vegetation isolines in red-nir reflectance space. Remote Sens. Environ. 74: 313-326. 